\title{
Lumbo-sacral epidural anaesthesia as a complement to dissociative anaesthesia during scrotal herniorrhaphy of livestock pigs in the field
}

\author{
Carl Ekstrand ${ }^{1,2 \dagger}$, Marie Sterning ${ }^{1}$, Love Bohman ${ }^{3}$ and Anna Edner ${ }^{1 *+}$
}

\begin{abstract}
Background: In Sweden, scrotal or inguinal herniorrhaphy of livestock pigs in the field has traditionally been an important part of the surgical skills training of veterinary students. Few substances meet the legal requirements for field anaesthesia of production animals in the European Union but a protocol based on azaperone-detomidinebutorphanol-ketamine does. Unfortunately the anaesthesia is characterised by unpredictable duration and depth and of abrupt awakenings which is not acceptable from an animal welfare perspective and impedes surgical training. Lumbo-sacral epidural analgesia is proven to provide sufficient analgesia to allow abdominal surgery, but there are few reports on the field use of this loco-regional technique. The study aim was to evaluate whether lumbo-sacral anaesthesia can be safely and successfully used in the field by a veterinary student and whether the combination of dissociative and lumbo-sacral epidural anaesthesia improves analgesia and anaesthesia to guarantee animal welfare during herniorrhaphy in livestock pigs, enabling surgical skills training.

Results: Pigs in the control-group (placebo) responded significantly stronger to surgery, with five out of 11 requiring additional doses of detomidine and ketamine. There were no significant differences between groups in respiratory rate, heart rate, blood pressure, $\mathrm{SpO}_{2}$ or blood gases. $\mathrm{SpO}_{2}$ levels $<94 \%$ were recorded in several pigs in both groups. No post-injection complications were reported at follow-up.

Conclusions: The results from this study showed that lumbo-sacral epidural anaesthesia with lidocaine could successfully be administered during dissociative anaesthesia of livestock pigs by a veterinary student and without reported post-injection complications. It improved analgesia and anaesthesia during herniorrhaphy of sufficient duration to enable surgical skills training. The risks and consequences of hypoxaemia and hypoventilation should be considered.
\end{abstract}

Keywords: Ketamine, Detomidine, Lumbo-sacral, Lidocaine, Surgery

\section{Background}

In the European Union (EU) only few substances are available for sedation and anaesthesia in livestock pigs. Regulations prescribe that all substances used need to be licensed for the species or authorised through the cascade principle $[1,2]$ and the substance has to be validated and listed in appendix I of the EU Commission regulation [3]. Azaperone, detomidine, butorphanol and

\footnotetext{
* Correspondence: anna.edner@slu.se

${ }^{\dagger}$ Equal contributors

'Department of Clinical Sciences, Faculty of Veterinary Medicine and Animal Science, Swedish University of Agricultural Sciences, PO box 7054 ,SE 75007 Uppsala, Sweden

Full list of author information is available at the end of the article
}

ketamine currently meet those requirements. Unfortunately, some pigs anaesthetised using a combination of these substances never reach a surgical plane of anaesthesia [4]. In addition, the anaesthesia is characterised by unpredictable duration and abrupt awakenings (Marie Sterning, SLU, Uppsala, Sweden, personal communication 2014). In Sweden, scrotal or inguinal herniorrhaphy of live-stock pigs in the field has traditionally been an important part of the surgical skills training of veterinary students. Due to the variable depth and length of dissociative anaesthesia when using the above listed, approved substances the number of pigs anesthetised in the field for surgical procedures to be performed by students has decreased. Thus, to ensure animal welfare 
during e.g. scrotal herniorrhaphy, the protocol needs modification.

Lumbo-sacral epidural administration of analgesic or local anaesthetic agents is widely used in hospital-based surgery in several species to improve intra- and postoperative analgesia of the abdomen and the hind limbs [5-8]. Local anaesthetics, for example lidocaine, administered epidurally provide desensitisation of the caudal abdomen and the hind limbs for up to $60 \mathrm{~min}$ [9]. In livestock pigs, lidocaine may be used according to the cascade principle or, in some countries, with a temporary general permit for all veterinarians. Publications of non-hospital use of lumbo-sacral epidural lidocaine in farm animals are limited [10]. To our knowledge, a protocol combining dissociative and lumbo-sacral epidural anaesthesia has not previously been evaluated in a field situation with livestock pigs.

The aims of the present study were to evaluate whether lumbo-sacral anaesthesia can be safely and successfully used in the field by a veterinary student and whether the combination of dissociative and lumbo-sacral epidural anaesthesia improves analgesia and anaesthesia to guarantee animal welfare during herniorrhaphy in livestock pigs enabling surgical skills training.

\section{Methods}

\section{Study design}

The study was designed as a prospective, randomised, blind experiment fulfilling all legal requirements for animal research in Sweden and was performed at a commercial pig-producing farm in Sweden. Approval was obtained from the Ethical Committee for Animal Research in Uppsala, Sweden (C154/9). A fifth-year veterinary student made all epidural injections and performed the surgery.

The owner was informed about the project, gave written consent for the farm's pigs to be used in the study and did not benefit financially from participating in the project.

\section{Pigs}

All 6-10-weeks-old, American Society of Anesthesiologist physical status I-II, non-castrated pigs with or without a scrotal hernia observed in the stables on study days were included in the study. The pigs were mixed breed Swedish Landrace-Yorkshire-Hampshire or Swedish LandraceYorkshire-Duroc with a mean weight (SD) of $14.3( \pm 3.4)$ $\mathrm{kg}$. They were randomly allocated (by picking coded notes from a jar) to either a group receiving an active treatment (epidural lidocaine, group L) or a control group receiving a non-active substance (epidural saline, group C). Field conditions were authentic in that daily routines such as feeding continued as usual and the pigs were not subjected to starvation before anaesthesia.

\section{Preparation for anaesthesia}

The pigs were visually inspected in order to detect obvious disease, which was cause for exclusion. Body weight of each pig was estimated and the pig was sedated with $4 \mathrm{mg} / \mathrm{kg}$ azaperone (Stresnil vet ${ }^{\circ}, 40 \mathrm{mg} / \mathrm{mL}$, Janssen Pharmaceutical, Belgium) intramuscularly (IM) in the neck muscle caudal to the base of the ear. The pig was left alone for $15 \mathrm{~min}$ in the pig house aisle with the lights switched off. It was then placed in a bag and weighed using a spring balance. If the bodyweight exceeded the estimated weight, the pig was given an additional injection of azaperone in order to reach $4 \mathrm{mg} / \mathrm{kg}$. Clinical examination including auscultation of heart and lungs, determination of heart rate (HR) and respiratory rate (RR), inspection of mucus membranes and determination of body temperature (BT) per rectum was performed in the sedated pig.

\section{Anaesthesia}

General anaesthesia was induced in the sedated pig immediately after the clinical examination. Detomidine $0.1 \mathrm{mg} / \mathrm{kg}$ (Domosedan vet $10 \mathrm{mg} / \mathrm{ml}$, Orion Pharma Animal Health, Sweden), butorphanol $0.2 \mathrm{mg} / \mathrm{kg}$ (Dolorex vet $^{\circ} 10 \mathrm{mg} / \mathrm{ml}$, Intervet, Sweden) and ketamine $10 \mathrm{mg} / \mathrm{kg}$ (Ketaminol vet ${ }^{\circ} 100 \mathrm{mg} / \mathrm{mL}$, Intervet, Sweden) were given separately IM in the neck muscle using a $0.6 \times 25 \mathrm{~mm}$ cannula. The pig also received an IM injection of ketoprofen $3 \mathrm{mg} / \mathrm{kg}$ (Romefen vet ${ }^{\circ} 100 \mathrm{mg} / \mathrm{mL}$, Merial Norden, Denmark) and was again left alone in the aisle. After $10 \mathrm{~min}$, the anaesthetised pig was transported to a small room within the pig house, where the epidural injection and surgery were performed. Pigs reacting to handling or surgery were given an additional injection of detomidine and ketamine preferentially intravenously (IV) but if this failed the IM route was used. If the reaction was exhibited before suturing the inguinal canal, $50 \%$ of the original dose was administered. A reaction after suturing the inguinal canal resulted in a bolus injection at $33 \%$ of the original dose, after which no surgical activity was performed for five minutes. If the pig still reacted to castration of the second testicle, analgesia was improved by infiltrating 20-40 mg $2 \%$ lidocaine intratesticularly.

The temperature in the temporary theatre varied between 19.9 and $24.6{ }^{\circ} \mathrm{C}$.

\section{Epidural injection}

The pig was placed in sternal recumbency on a metal table covered with a folded cloth, with the hips flexed and the hind legs extended alongside the abdomen. The length of the vertebral column was measured from the external occipital process to the first vertebra of the tail in order to calculate the doses for epidural injection [11] $1 \mathrm{ml}$ for the first $40 \mathrm{~cm}$ of vertebral column, and another $0.15 \mathrm{ml}$ for each additional $\mathrm{cm}$. The lumbo-sacral area 
was aseptically prepared, although the hair was not clipped. Wearing sterile gloves, a needle (BD Microlance $^{\text {Tx }} \quad 3, \quad 0.9 \mathrm{~mm} \times 40 \mathrm{~mm}$, BectonDickinson and Company, USA) was placed in the extradural space at a $90^{\circ}$ angle to the back between the last lumbar vertebrae and the first sacral vertebrae. Correct placement was confirmed when no blood or liquor was obtained on aspiration and loss of resistance occurred on test-injecting saline.

In group L, 2 \% lidocaine (Xylocain ${ }^{\circ}$, AstraZeneca, Sweden) was administered and group $\mathrm{C}$ received physiological saline. The injected volume was delivered over one minute. The containers of both substances were masked identically and not identifiable to the anaesthesiologist or the surgeon.

\section{Surgery}

One minute after the epidural injection, the pig was turned into dorsal recumbency and the inguinal area prepared for surgery. Five minutes after the epidural injection, an approximately $10 \mathrm{~cm}$ long skin incision was made in the groin, above the inguinal canal. After manually detaching the testicle from the scrotum, the intestines were repositioned back into the abdomen. The spermatic cord was torqued, clamped with a haemostat and ligated with a transfixed ligature $\left(\right.$ Vetafil $^{\circ} 0.4 \mathrm{~mm}$, WDT, Germany). At this point the hind end of the table was raised from horizontal to $10^{\circ}$ to prevent the intestines from falling back into the hernia. The inguinal canal was closed with a continuous suture (Vetafil $^{\circ}$ $0.4 \mathrm{~mm}$ ) and then the short end of the table was replaced in its original position and the skin was sutured with 2-3 isolated stitches (Vetafil $0.3 \mathrm{~mm}$, WDT, Germany). Surgery followed a specific time schedule, to mimic a possible surgical skills training event by a fifthyear veterinary student (see below).

Immediately after suture of the skin closed castration of the contra-lateral side was performed. The spermatic cord was ligated using a transfixed ligature (Vetafil ${ }^{\circ} 0.4 \mathrm{~mm}$ ). The castration was performed without intermission. The wound was left for second intention healing.

If having a scrotal hernia this was repaired on the affected side and routine castration was performed on the unaffected side. If the pig was an intact male without a hernia, castration of one side was performed using the scrotal hernia surgery technique.

Time schedule for the procedure:

- -10 min (T-10): Preparation for epidural injection, ventral recumbency.

- $-5 \min (\mathrm{T}-5)$. Epidural injection.

- -3 min (T-3). After epidural injection, after being turned to dorsal recumbency.

- 0 min (T0). Skin incision.
- 7 min (T7). Detachment of testis from scrotal wall.

- 10 min (T10). Clamping of spermatic cord. Table hind end elevated.

- 12 min (T12). Ligation of spermatic cord. Table hind end elevated.

- 17 min (T17). Suture of inguinal canal. Table hind end lowered after suture.

- 30 min (T30). Suture of the skin.

- Castration of other testicle, uninterrupted procedure.

Bleeding control after castration served as endpoint for surgery.

After surgery, the pig was marked in the ear for postoperative identification by the farmer and was left to recover in a small straw-filled carriage. When able to walk, it was returned to the aisle of its pen, where it stayed until the day after surgery before being returned to its home pen.

\section{Collection of data}

Heart rate, RR, nociceptive responses (pedal withdrawal reflex, PWR; nasal septum pinch, NSP), sensitivity of the snout, palpebral reflex, nystagmus and muscular relaxation (jaw and forelimbs) were monitored manually by an experienced anaesthesiologist from preparation for epidural injection (T-10) until completion of surgery. PWR and NSP were tested using a haemostat maximally clamped to the first hatchet. Sensitivity of the snout was tested by gently stroking the snout with the index finger.

Reaction to surgery (change in breathing pattern, vocalisation or motions) was continuously monitored. The reactions were graded as positive or negative.

During the procedure, non-invasive systolic, diastolic and median blood pressure (SAP, DAP, MAP) was measured oscillometrically using a cuff placed above the carpus of a forelimb (Cardell ${ }^{\circ}$ Veterinary Monitor 9402, Sharn Veterinary Inc. Tampa, Florida, USA). A pulse oximetry probe was placed on one of the pig's ears or tongue and connected to the same machine as for blood pressure (BP). Physiological variables are reported at 5-min intervals from T-10 to T35.

At T5 and T30, arterial blood was collected anaerobically from 8 pigs in group $C$ and 7 pigs in group L. The samples were processed immediately using a portable analyser and cartridges (i-STAT ${ }^{\circ} 1$ Portable Clinical Analyzer, cartridge CG4+, Abbott Laboratories, Illinois, USA). Analyses included measurement of partial pressure of arterial oxygen $\left(\mathrm{PaO}_{2}\right)$ and carbon dioxide $\left(\mathrm{PaCO}_{2}\right)$ and $\mathrm{pH}$, and calculated values of haemoglobin oxygen saturation $\left(\mathrm{SaO}_{2}\right)$ at $37{ }^{\circ} \mathrm{C}$. Body temperature was measured rectally in the azaperone-sedated pig and at the end of surgery, using a digital thermometer. Time 
for recovery to sternal recumbency and standing was only roughly noted.

\section{Interpretation of data}

On responding positively to a stimulus with a dichotomous outcome, the pig was registered as a responder and thereafter considered a responder for the remainder of the procedure. This type of registration is denoted accumulated frequency'. Hypoxaemia was defined as a $\mathrm{SpO}_{2}<90 \%$.

\section{Statistics}

Statistical analyses were carried out on all measured variables. For variables with a count outcome (nociceptive responses, sensitivity of the snout, palpebral reflex, nystagmus, muscular relaxation and iteration), Fisher's exact test (1-sided) was employed. Body temperature, body weight, $\mathrm{PaO}_{2}, \mathrm{PaCO}_{2}, \mathrm{pH}$, surgery time and time to recovery were analysed with t-tests (2-sided).

Variables with a continuous outcome (DAP, MAP, SAP and $\mathrm{SpO}_{2}$ ) were analysed with repeated measurement ANOVA. In addition, differences between the treatment group (L) and the control group (C) were tested at each timepoint by Šidák-adjusted t-test. Šidák adjustment was also employed for within-group comparisons between each timepoint and the value at T0 (start of surgery).
Values were considered significant at $P<0.05$. Results are presented as mean \pm standard deviation (SD). All statistical calculations were performed using Stata 12.1 (StataCorp. 2011. Stata Statistical Software: Release 12. College Station, TX: StataCorp LP, Texas, USA).

\section{Results}

A total of 22 pigs were included in the study, with 11 pigs each in groups $C$ and $L$. The average weight was $14.1 \pm 4.7 \mathrm{~kg}$ in group $\mathrm{C}$ and $14.6 \pm 1.5 \mathrm{~kg}$ in group L, with no difference between groups. The volumes administered epidurally were $0.28 \pm 0.02 \mathrm{ml} / \mathrm{kg}$ in group C and $0.29 \pm 0.02 \mathrm{ml} / \mathrm{kg}$ in group L, equivalent to a dose of $5.7 \pm$ $0.5 \mathrm{mg} / \mathrm{kg}$. Time from administration of ketamine until first incision was $28 \pm 1 \mathrm{~min}$ and $27 \pm 3 \mathrm{~min}$ in group $\mathrm{C}$ and $\mathrm{L}$, respectively.

There were significant differences between the groups in regard to the accumulated frequency of the variables PWR, NSP, muscular relaxation of forelimbs, spontaneous movements and reaction to surgery with more responders in group C (Fig. 1). No pig in group L reacted to surgery during herniorrhaphy, whereas three group $\mathrm{C}$ pigs reacted to surgery on more than one occasion. One group C pig and one group L pig reacted at castration of the second testicle (not shown in Fig. 1). The pig in

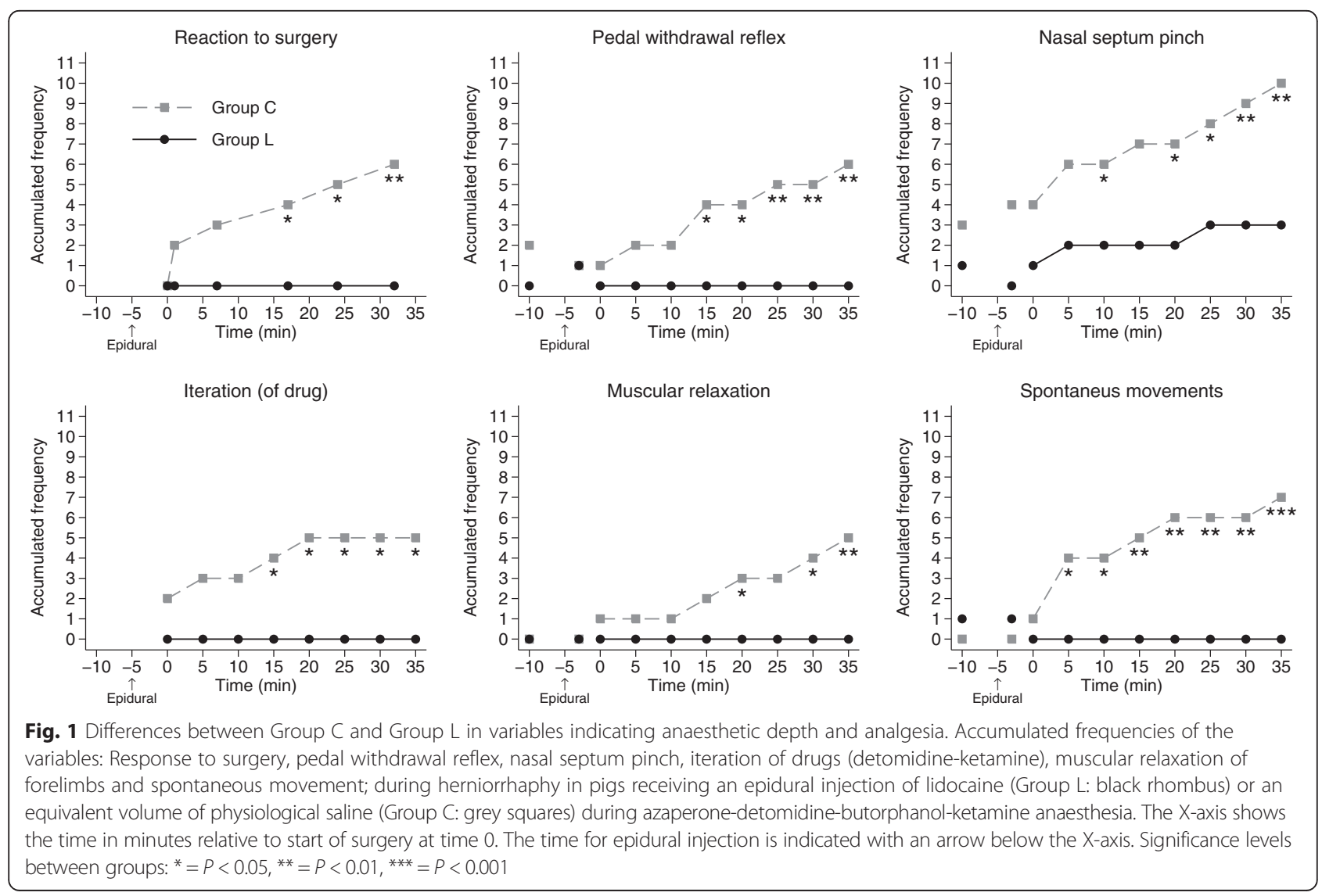


group L only showed a minor, transient change in breathing pattern.

The variables nystagmus, palpebral reflex and snout sensibility did not differ between the groups.

In group $\mathrm{C}$ five pigs received one additional injection of detomidine and ketamine and one pig received two additional injections, at $50 \%$ of the initial dose, due to response to surgery. No pig in group L received an extra dose of anaesthetics. Three pigs in group $\mathrm{C}$ were administered lidocaine locally to enable completion of surgery, two of which had previously received additional ketamine-detomidine. No pig in group L received local administration of lidocaine. Significantly more pigs in group $\mathrm{C}$ received additional ketamine-detomidine and/ or local lidocaine $(P<0.01)$.

Body temperature decreased similarly in both groups from pre-anaesthesia to post-surgery, from $38.8 \pm 0.4{ }^{\circ} \mathrm{C}$ to $36.1 \pm 1.0{ }^{\circ} \mathrm{C}$ in group $\mathrm{C}(P<0.001)$ and from $39.1 \pm$ $0.5{ }^{\circ} \mathrm{C}$ to $36.8 \pm 1.0^{\circ} \mathrm{C}$ in group $\mathrm{L}(P<0.001)$.

Heart rate, RR, SAP, MAP and DAP did not differ between the groups (Figs. 2 and 3). Mean arterial blood pressure was $<60 \mathrm{mmHg}$ during at least half of the procedure in two of the 11 pigs in group $\mathrm{C}$ and in four of the 11 pigs in group L.

There was a tendency for a general difference between groups as regards $\mathrm{SpO}_{2}(P=0.06)$, but after Šidák correction the post hoc analysis could not identify any specific timepoint at which the mean values differed (Fig. 3). $\mathrm{SpO}_{2}$ values below $90 \%$ at one or more sampling points were registered in $5 / 11$ and $7 / 11$ pigs in Group C and L respectively, and at 16 out of 113 sampling points in group $C$ and at 44 out of 115 sampling points in Group L. Between T-10 and T0, 3/11 pigs in group $\mathrm{C}$ and $8 / 11$ pigs in group $\mathrm{L}$ showed decreasing values for $\mathrm{SpO}_{2}$, but no significant changes over time were recorded in either group.
Mean values $\left( \pm\right.$ SD) for $\mathrm{PaO}_{2}, \mathrm{PaCO}_{2}, \mathrm{SaO}_{2}$ and $\mathrm{pH}$ are shown in Table 1 . Paired arterial samples were only successfully obtained and analysed in five pigs in group C and in seven pigs in group L. Mean values were calculated on all samples, but statistics over time were only performed on paired samples. There were no significant differences in $\mathrm{PaO}_{2}$ or $\mathrm{PaCO}_{2}$ between groups. $\mathrm{PaO}_{2}$ decreased over time in group $\mathrm{L}(P=0.02)$ but not in group $\mathrm{C}(P=0.94)$. A $\mathrm{PaO}_{2} \leq 8 \mathrm{kPa}$ (and $\left.\mathrm{SaO}_{2} \leq 90 \%\right)$ was measured in 3/13 samples from group $C$ and in $4 / 14$ samples from group L. A small but statistically significant increase was observed in $\mathrm{PaCO}_{2}$ in group $\mathrm{C}$ $(P=0.02)$. No difference over time was detected in group L $(P=0.25)$. There was a significant difference in arterial $\mathrm{pH}$ between groups at both sampling timepoints $(p<0.01$ at T5 and $P=0.03$ at T30), but there was no significant change over time within groups.

Total surgery time (from incision until end of castration) was $41 \pm 3 \mathrm{~min}$ in group $\mathrm{C}$ and $39 \pm 5 \mathrm{~min}$ in group $\mathrm{L}$. The approximate time from end of surgery until the pig was able to run was $76 \pm 33 \mathrm{~min}$ in group $C(n=10)$ and $87 \pm 33 \mathrm{~min}$ in group L $(n=9)$. No statistical comparison was performed on recovery times since the exact recovery times were not recorded.

All pigs recovered uneventfully from surgery and were returned to their home pens on the morning after surgery. No complication associated with surgery or the epidural injection was reported by the farmer at followup.

\section{Discussion}

Comparing the frequency of intra-operative responses to noxious stimuli in the present study, dissociative anaesthesia combined with epidural administration of lidocaine was superior to dissociative anaesthesia alone during field surgery in pigs. It provided additional

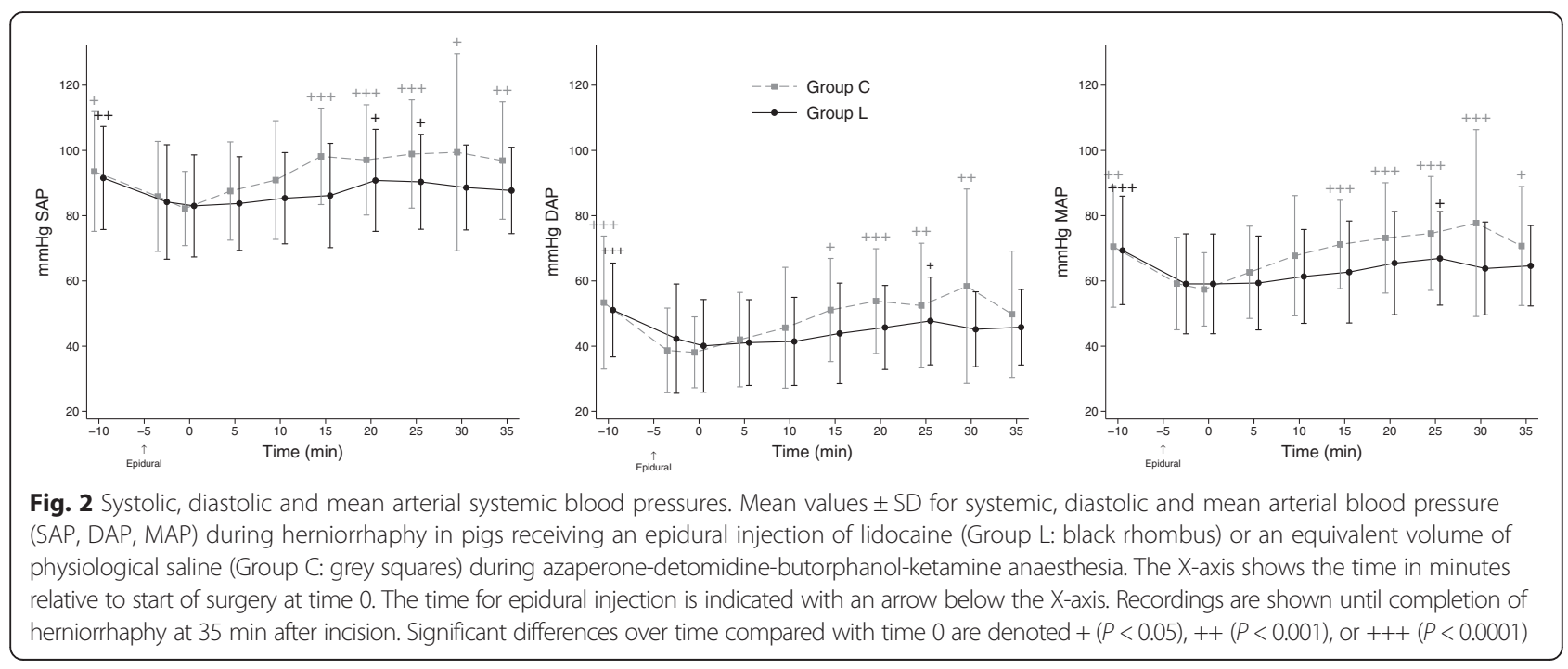



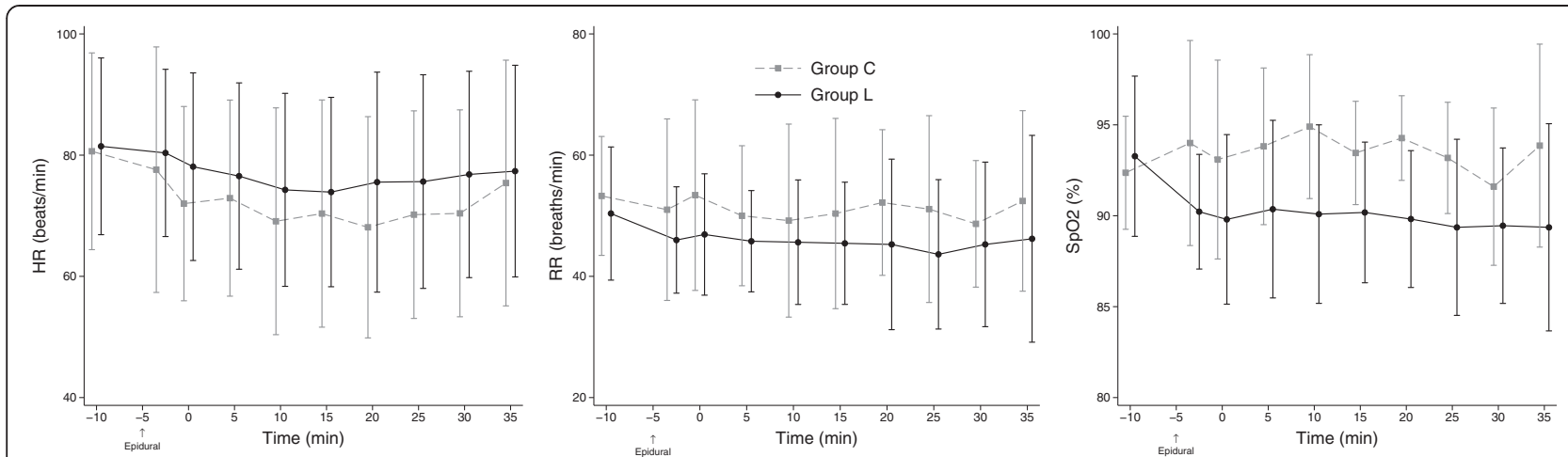

Fig. 3 Heart rate, respiratory rate and $\mathrm{SpO}_{2}$. Mean values $\pm \mathrm{SD}$ for heart rate (HR), respiratory rate (RR) and $\mathrm{SpO}_{2}$ during herniorrhaphy in pigs receiving an epidural injection of lidocaine (group L: black rhombus) or an equivalent volume of physiological saline (group C: grey squares) during azaperone-detomidine-butorphanol-ketamine anaesthesia. The $\mathrm{X}$-axis shows the time in minutes relative to start of surgery at time 0.

The time for epidural injection is indicated with an arrow below the $X$-axis. Recordings are shown until completion of herniorrhaphy at 35 min after incision. Significant differences over time compared with time 0 are denoted $+(P<0.05)$, $++(P<0.001)$, or $+++(P<0.0001)$

analgesia of sufficient degree and duration to guarantee animal welfare during scrotal herniorrhaphy and no postinjection complications were reported. It also proved a functional protocol for a teaching situation in which veterinary students practise their surgical skills. In the tested group no further clinically significant negative effects on the cardiovascular system were registered, although the results indicated a slightly more negative effect on gas exchange compared with dissociative anaesthesia alone. Thus the protocol appears to provide acceptable safety margins for field surgery in livestock pigs.

Seven out of 11 pigs in the control group reacted to surgery, during a surgical procedure shorter than $40 \mathrm{~min}$. Among those pigs, two reacted at the initial

Table 1 Partial pressures of $\mathrm{O}_{2}$ and $\mathrm{CO}_{2}$, saturation of oxygen and $\mathrm{pH}$ in arterial blood

\begin{tabular}{llll}
\hline & & $l$ & \\
Variable & Group & T5 & T30 \\
\hline $\mathrm{PaO}_{2}(\mathrm{kPa})$ & $\mathrm{C}$ & $9.6 \pm 2.2$ & $10.1 \pm 1.9$ \\
& $\mathrm{~L}$ & $10.0 \pm 1.7$ & $8.5 \pm 1.9$ \\
$\mathrm{PaCO}_{2}(\mathrm{kPa})$ & $\mathrm{C}$ & $6.2 \pm 1.0$ & $6.3 \pm 0.6$ \\
& $\mathrm{~L}$ & $6.8 \pm 0.5$ & $7.2 \pm 1.0$ \\
$\mathrm{SaO}_{2}(\%)$ & $\mathrm{C}$ & $92 \pm 6$ & $94 \pm 3$ \\
& & $94(82-97)$ & $95(88-97)$ \\
& $\mathrm{L}$ & $92 \pm 3$ & $87 \pm 10$ \\
& & $92(88-96)$ & $93(68-95)$ \\
$\mathrm{pH}$ & $\mathrm{C}$ & $7.38 \pm 0.04$ & $7.38 \pm 0.03$ \\
& $\mathrm{~L}$ & $7.32 \pm 0.01^{* *}$ & $7.32 \pm 0.05^{*}$ \\
\hline
\end{tabular}

Mean values ( \pm SD) at 5 and 30 min after start of surgery (T5 and T30) in control pigs (group $C, n=8$ ) and in pigs receiving epidural lidocaine (group $L$, $n=7$ ) during azaperone-detomidine-butorphanol-ketamine anaesthesia. $\mathrm{PaO}_{2}$ : partial pressure of arterial oxygen; $\mathrm{PaCO}_{2}$ : partial pressure of arterial carbon dioxide; $\mathrm{SaO}_{2}$ : arterial oxygen saturation of haemoglobin. Significant differences between groups: ${ }^{*}=P<0.05 ;{ }^{* *}=P<0.01$. Significant differences to T5 are denoted $(=P<0.05)$ incision of the skin and were obviously not at a level of anaesthesia profound enough even for superficial surgery. Using a similar protocol as for the control group in the present study, Heinonen et al. [4] concluded that dissociative anaesthesia was unreliable in pigs, but can be used for minor surgery. In that study, one-third of the pigs never reached a surgical level of anaesthesia and the duration of anaesthesia showed great individual variation. In contrast, a protocol composed of medetomidine-ketamine-butorphanol resulted in excellent surgical conditions [12]. However, medetomidine is not available for use in production animals in the European Union. Lumbo-sacral epidural anaesthesia is frequently used in in-hospital surgical procedures to provide analgesia of the caudal abdomen and was therefore added to the anaesthesia protocol approved for livestock pigs. The single pig in group L that reacted to surgery did so $45 \mathrm{~min}$ after epidural injection, when the effect of lidocaine had most likely begun to wear off. The reported duration of epidural anaesthesia using lidocaine in pigs varies between 30 and $60 \mathrm{~min}[9,13]$. The present protocol was developed to mimic a situation where final-year veterinary students perform herniorrhaphy as part of their surgical training. Therefore, the timing of events was slower than if surgery had been performed by an experienced veterinarian. Despite this, surgical conditions were excellent for most of the procedure in group L. In a routine situation, when a skilled veterinarian performs hernia surgery, it is usually completed within $20 \mathrm{~min}$, so the duration of epidural lidocaine is sufficient in most circumstances.

In the present study, apart from reducing the nociceptive stimuli from the surgery site, epidural lidocaine also enhanced the depth of anaesthesia, as indicated by the decreased response to nociceptive provocations of the 
forelimbs (PWR) and nasal septum (NSP). Previous studies have shown that epidural analgesia/anaesthesia reduces the requirement for anaesthetic or hypnotic agents in several species such as pigs [14], dogs [15, 16], horses [7] and humans [17-19]. This effect might be explained by indirect central effects of spinal deafferentation $[19,20]$.

Despite the obvious differences between groups in the physical response to noxious stimuli, no differences in BP, HR or RR were detected between groups, and timerelated changes in BP were similar in both groups. Increases in BP and HR are commonly used as indicators of intra-operative nociception [21, 22]. However, since the groups were small and blood pressure only measured oscillometrically, minor differences in BP and HR may not have been detected.

Several pigs in both groups of the present study were hypoxaemic $\left(\mathrm{SpO}_{2}\right.$ or $\left.\mathrm{SaO}_{2}<90 \%\right)$ at one or more sampling points during dissociative anaesthesia. The mean values for $\mathrm{PaO}_{2}$ in this study were lower than in the medetomidine-ketamine-butorphanol anaesthetised pigs [12], but comparable to the values presented by Heinonen et al. [4]. The pigs in the present study were positioned in dorsal recumbency, in contrast to the laterally recumbent pigs in the study by Sakaguchi et al. [12], which may have contributed to hypoxaemia [23, 24]. In addition, these pigs were livestock pigs, in which subclinical disease may have been present, possibly affecting oxygen uptake. Hypoxaemia is a well-recognised sequel to anaesthesia, and therefore oxygen supplementation is often standard routine during in-hospital procedures in anaesthetised or deeply sedated animals. In the field, oxygen may be provided through an oxygen cylinder or an oxygen generator and delivered through a face mask. However, for economic reasons this is not commonly practised in Sweden. To mimic the field situation, oxygen was not administered to the pigs in the present study.

Epidural anaesthesia using lidocaine has been reported to induce depression of respiratory function, with increases in $\mathrm{PaCO}_{2}$ and decreases in $\mathrm{PaO}_{2}[9$, 25]. However, in the present study, on the basis of the few arterial samples taken, hypoventilation was similar in both groups. It is possible that with the inclusion of more animals and analysis of more arterial blood gas samples, significant group differences would have been detected. A similar degree of hypoventilation during dissociative anaesthesia of pigs has been reported previously [4, 12]. Arterial blood $\mathrm{pH}$ was lower in group $\mathrm{L}$ but the lowest individual $\mathrm{pH}$ values were not consistently associated with the highest $\mathrm{PaCO} 2$ values, which may indicate a metabolic component of the acidaemia. Since plasma lactate concentration rise quickly in pigs during stress
[26], such as caused by handling, this may have contributed to the low $\mathrm{pH}$.

The pigs recovered well from their lidocaine-induced paresis and no pig showed prolonged loss of motor function beyond recovery from dissociative anaesthesia. Epidural injections are usually performed under sterile conditions to reduce the risk of introducing bacteria into the spinal canal. Despite the non-sterile conditions in the present study, no post-injection infections were reported for any of the 22 pigs. Detailed knowledge of anatomy and careful attention to correct placement of the needle also reduce the risk of complications. The results from this and another field study [10] indicate that the lumbosacral injection technique can be successfully used in a field situation.

\section{Conclusion}

Based on the results of the present study, epidural administration of lidocaine can be recommended in pigs anaesthetised with azaperone-detomidine-butorphanolketamine, in order to improve the quality of anaesthesia and analgesia during field surgery and during surgical skills training by veterinary students. However, the pigs are at risk of developing hypoxaemia and provision of supplemental oxygen should be considered.

\section{Competing interests}

The authors declare that they have no competing interests.

\section{Authors' contributions}

$C E$, and AE planned and performed the study, interpreted data and drafted the manuscript. MS planned and performed the study, coordinated contacts between the research group and the farm and revised the manuscript. LB performed the statistical analyses, drafted relevant sections of the manuscript and revised rest of the manuscript. All authors read and approved the final manuscript.

\section{Acknowledgements}

The authors wish to express their sincere gratitude to SLU for financial support (Gymnastikdirektör Stina Johanssons i Fränsta fond för veterinärmedicinsk forskning and Regementsveterinär och fru Carl Ahlgrens stipendiefond) and to the owners and staff at Nygård pig farm where the project was conducted.

\section{Author details}

${ }^{1}$ Department of Clinical Sciences, Faculty of Veterinary Medicine and Animal Science, Swedish University of Agricultural Sciences, PO box 7054, SE 75007 Uppsala, Sweden. ²Department of Biomedicine and Veterinary Public Health, Faculty of Veterinary Medicine and Animal Science, Swedish University of Agricultural Sciences, PO box 7028SE 75007 Uppsala, Sweden. ${ }^{3}$ Department of Sociology, Stockholm University, SE 10691 Stockholm, Sweden.

Received: 29 April 2015 Accepted: 17 June 2015

Published online: 24 June 2015

\section{References}

1. EC. Directive 2001/82/EC of the European Parliament and of the Council of 6 November 2001 on the Community code relating to veterinary medicinal products. (Official Journal L 311, 28/11/2001 p. 1 - 66). (consolidated version: 18/7/2009). 2001 [http://ec.europa.eu/health/documents/eudralex/vol-5/ index_en.htm].

2. EC. Directive 2004/28/EC of the European Parliament and of the Council of 31 March 2004 amending Directive 2001/82/EC on the Community code 
relating to veterinary medicinal products (Official Journal L 136, 30/4/2004 p. 58 - 84). 2004 [http://ec.europa.eu/health/documents/eudralex/vol-5/ index_en.htm].

3. EU. Comission Regulation (EU) No 37/2010 of 22 December 2009 on pharmacologically active substances and their classification regarding maximum residue limits in foodstuffs of animal origin Official Journal of the European Union L 15/1 II. 2010 [http://ec.europa.eu/health/files/eudralex/ vol-5/reg_2010_37/reg_2010_37_en.pdf].

4. Heinonen ML, Raekallio MR, Oliviero C, Ahokas S, Peltoniemi OA. Comparison of azaperone-detomidine-butorphanol-ketamine and azaperone-tiletamine-zolazepam for anaesthesia in piglets. Vet Anaesth Analg. 2009;36:151-7.

5. Malavasi LM, Jensen-Waern M, Jacobson M, Ryden A, Ohagen P, Nyman G. Effects of extradural morphine on end-tidal isoflurane concentration and physiological variables in pigs undergoing abdominal surgery: a clinical study. Vet Anaesth Analg. 2006;33:307-12.

6. Almeida RM, Escobar A, Maguilnik S. Comparison of analgesia provided by lidocaine, lidocaine-morphine or lidocaine-tramadol delivered epidurally in dogs following orchiectomy. Vet Anaesth Analg. 2010;37:542-9.

7. Doherty TJ, Geiser DR, Rohrbach BW. Effect of high volume epidural morphine, ketamine and butorphanol on halothane minimum alveolar concentration in ponies. Equine Vet J. 1997;29:370-3.

8. Stegmann GF. Cardiovascular effects of lumbar epidural anaesthesia in isoflurane-anaesthetised pigs during surgical removal of the liver. I S Afr Vet Assoc. 2009:80:37-40

9. Tendillo FJ, Pera AM, Mascias A, Santos M, Desegura IAG, Roman FS, et al. Cardiopulmonary and analgesic effects of epidural lidocaine, alfentanil, and xylazine in pigs anesthtized with isoflurane. Vet Surg. 1995;24:73-7.

10. Scott PR, Sargison ND, Penny CD, Pirie RS. Application of lumbosacral spinal anesthesia for ovine Caesarian surgery and for vasectomy under field conditions. Theriogenology. 1994;42:891-3.

11. Strande A. Epidural anaesthesia in young pigs, dosage in relation to the length of the vertebral column. Acta Vet Scand. 1968;9:41-9.

12. Sakaguchi M, Nishimura R, Sasaki N, Ishiguro T, Tamura H, Takeuchi A. Anesthesia induced in pigs by use of a combination of medetomidine, butorphanol, and ketamine and its reversal by administration of atipamezole. Am J Vet Res. 1996;57:529-34.

13. Paavola A, Tarkkila P, Xu M, Wahlstrom T, Yliruusi J, Rosenberg P. Controlled release gel of ibuprofen and lidocaine in epidural use-analgesia and systemic absorption in pigs. Pharm Res. 1998;15:482-7.

14. Malavasi LM, Jensen-Waern M, Augustsson H, Nyman G. Changes in minimal alveolar concentration of isoflurane following treatment with medetomidine and tiletamine/zolazepam, epidural morphine or systemic buprenorphine in pigs. Lab Anim. 2008:42:62-70.

15. Valverde A, Dyson DH, McDonell WN. Epidural morphine reduces halothane MAC in the dog. Can J Anaesth. 1989;36:629-32.

16. Tusell JM, Andaluz A, Prandi D, Costa C, Garcia F. Effects of epidural anaesthesia-analgesia on intravenous anaesthesia with propofol. Vet J. 2005;169:108-12.

17. Schwieger IM, Klopfenstein CE, Forster A. Epidural morphine reduces halothane MAC in humans. Can J Anaesth. 1992;39:911-4.

18. Tverskoy M, Shifrin V, Finger J, Fleyshman G, Kissin I. Effect of epidural bupivacaine block on midazolam hypnotic requirements. Reg Anesth. 1996;21:209-13.

19. Hodgson PS, Liu SS, Gras TW. Does epidural anesthesia have general anesthetic effects? A prospective, randomized, double-blind, placebocontrolled trial. Anesthesiology. 1999;91:1687-92.

20. Hodgson PS, Liu SS. Epidural lidocaine decreases sevoflurane requirement for adequate depth of anesthesia as measured by the Bispectral Index monitor. Anesthesiology. 2001;94:799-803.

21. Moldal ER, Eriksen T, Kirpensteijn J, Nodtvedt A, Kristensen AT, Sparta FM et al. Intratesticular and subcutaneous lidocaine alters the intraoperative haemodynamic responses and heart rate variability in male cats undergoing castration. Vet Anaesth Analg. 2013;40:63-73.

22. Huuskonen V, Hughes JM, Estaca Banon E, West E. Intratesticular lidocaine reduces the response to surgical castration in dogs. Vet Anaesth Analg. 2013;40:74-82.

23. Niehues SM, Muller C, Plendl J, Richardson KC, Gemeinhardt O, Hunigen $\mathrm{H}$, et al. The effect of prone versus supine positioning of Goettingen minipigs on lung density as viewed by computed tomography. Clin Hemorheol Microcirc. 2012;52:85-92.
24. Altemeier WA, McKinney S, Krueger M, Glenny RW. Effect of posture on regional gas exchange in pigs. J Appl Physiol (Bethesda, Md: 1985). 2004;97:2104-11.

25. Mpanduji DG, Mgasa MN, Bittegeko SB, Batamuzi EK. Comparison of xylazine and lidocaine effects for analgesia and cardiopulmonary functions following lumbosacral epidural injection in goats. Zentralbl Veterinarmed A. 1999:46:605-11.

26. Jensen-Waern $M$, Nyberg L. Valuable indicators of physical stress in porcine plasma. Zentralbl Veterinarmed A. 1993;40:321-7.

\section{Submit your next manuscript to BioMed Central and take full advantage of:}

- Convenient online submission

- Thorough peer review

- No space constraints or color figure charges

- Immediate publication on acceptance

- Inclusion in PubMed, CAS, Scopus and Google Scholar

- Research which is freely available for redistribution 